\title{
Conformally flat 3-manifolds with constant scalar curvature II
}

\author{
By Qing-Ming Cheng*, Susumu Ishikawa** and Katsuhiro Shiohama** \\ (Received November 13, 1998) \\ (Revised October 5, 2000) \\ (from Kyushu Journal of Mathematics)
}

\section{Introduction}

A well known result in Riemannian geometry states that every compact $n$ dimensional Riemannian manifold can be deformed to a Riemannian manifold with constant scalar curvature by a conformal transformation (see [3], [5], [6], etc.). This result was obtained through the application of the so-called Yamabe equation. The Yamabe equation has a deep connection with locally conformally flat Riemannian manifolds (see [4]). Let us begin by recalling that $M^{n}$ is by definition locally conformally flat if and only if every point on $M^{n}$ admits a coordinate neighborhood with coordinates $u^{1}, \ldots, u^{n}$ in which the Riemannian metric can be expressed as

$$
d s^{2}=\sum_{i=1}^{n} \lambda^{2}(u)\left(d u^{i}\right)^{2}
$$

where $\lambda(u)$ is a positive function defined in the coordinate neighborhood. There are many examples of locally conformally flat Riemannian manifolds (see [4], etc.).

The study of global properties on locally conformally flat Riemannian manifolds was initiated by Kuiper, who studied their fundamental groups. Recently, Schoen and Yau also investigated the global properties on the locally conformally flat Riemannian manifolds by using the Yamabe equation. In the previous paper [1], we have classified in dimension 3 the class of such Riemannian manifolds with a certain additional properties. Let $r$ and $S$ be respectively the scalar curvature and the squared norm of Ricci curvature tensor of a complete locally conformally

\footnotetext{
* Research partially Supported by the Grant-in-Aid for Scientific Research from the Ministry of Education, Science, Sports and Culture and by the Grant-in-Aid for Scientific Research from Josai University.

${ }^{* *}$ Research partially Supported by the Grant-in-Aid for Scientific Research from the Ministry of Education, Science, Sports and Culture.
} 
flat $n$-dimensional Riemannian manifold $M^{n}$. Let $M^{n}(c)$ be $n$-dimensional space form of constant sectional curvature $c$. The following results have been established in [1]:

Let $M^{3}$ be a complete locally conformally flat 3-dimensional Riemannian manifold with both $r$ and $S$ being constant. If $r \geq 0$, then

(1) $M^{3}$ is isometric to a space form of constant sectional curvature

or

(2) $\quad M^{3}$ is isometric to a Riemannian product $M^{2}(c) \times N^{1}$ where $N^{1}=\mathbf{R}^{1}$ or $S^{1}$ for some $c \geq 0$.

If $r<0$, then

(3) $M$ is isometric to a space form of constant negative curvature

or

(4) $\frac{r^{2}}{3}<S \leq \frac{r^{2}}{2}$.

We have also proved in [1] that there exists no compact locally conformally flat Riemannian 3-manifold $M^{3}$ with the following two conditions:

(a) $r$ is a negative constant

(b) $S$ is constant and all eigenvalues of the Ricci curvature tensor at each point of $M^{3}$ are distinct.

Typical examples are stated as follows.

EXAMPLE 1. $H^{3}(r / 6)(r<0)$ is a complete locally conformally flat 3-dimensional Riemannian manifold with $S=\frac{r^{2}}{3}$.

EXAMPle 2. $H^{2}(c) \times \mathbf{R}(c<0)$ is a complete locally conformally flat 3dimensional Riemannian manifold with $S=\frac{r^{2}}{2}$.

However, it has not yet been known if there exists a complete locally conformally flat 3-dimensional Riemannian manifold on which $r$ is a negative constant and $S$ is constant such that $\frac{r^{2}}{3}<S<\frac{r^{2}}{2}$. The following conjecture has been proposed in [1]

CONJECTURE. Let $M^{3}$ be a complete locally conformally flat 3-dimensional Riemannian manifold with the constant negative scalar curvature and the Ricci curvature tensor of constant squared norm. Then, $M^{3}$ is isometric to either a space form of constant negative curvature or the Riemannian product $H^{2}(c) \times N^{1}(c<0)$.

The purpose of present article is to study complete locally conformally flat 3dimensional Riemannian manifolds with constant negative scalar curvature $r$ possessing Ricci curvature tensor of constant squared norm $S$. Our Main Theorem strongly supports the validity of the above conjecture. 
MaIn Theorem. There exist no complete locally conformally flat 3-dimensional Riemannian manifolds with negative constant scalar curvature $r$ and the Ricci curvature tensor with constant squared norm $S$ satisfying $\frac{r^{2}}{3}+\left(\frac{7}{113}\right)^{2} \frac{2 r^{2}}{3}<$ $S<\frac{r^{2}}{3}+\left(\frac{35}{137}\right)^{2} \frac{r^{2}}{6}$.

Acknowledgement. Authors would like to express their gratitude to the referee for his valuable suggestion.

\section{Preliminaries}

Let $M^{n}$ be an $n$-dimensional Riemannian manifold. We choose a local frame of orthonormal vector fields $\left\{e_{1}, \ldots, e_{n}\right\}$ adapted to the Riemannian metric of $M^{n}$ and the dual coframe $\left\{\omega_{1}, \ldots, \omega_{n}\right\}$. Then the connection forms $\left\{\omega_{i j}\right\}$ of $M^{n}$ are characterized by the structure equations:

$$
\begin{gathered}
d \omega_{i}=-\sum_{j} \omega_{i j} \wedge \omega_{j}, \quad \omega_{i j}+\omega_{j i}=0, \\
d \omega_{i j}=-\sum_{k} \omega_{i k} \wedge \omega_{k j}+\frac{1}{2} \sum_{k, l} R_{i j k l} \omega_{k} \wedge \omega_{l},
\end{gathered}
$$

where $R_{i j k l}$ are the components of curvature tensor of $M^{n}$.

Let $C_{i j k l}$ denote the components of Weyl tensor of $M^{n}$, that is,

$$
\begin{aligned}
C_{i j k l}= & R_{i j k l}-\frac{1}{n-2}\left(R_{i k} \delta_{j l}-R_{i l} \delta_{j k}+R_{j l} \delta_{i k}-R_{j k} \delta_{i l}\right) \\
& +\frac{r}{(n-1)(n-2)}\left(\delta_{i k} \delta_{j l}-\delta_{i l} \delta_{j k}\right)
\end{aligned}
$$

where $R_{i j}$ and $r$ are the components of Ricci curvature tensor and the scalar curvature of $M^{n}$, respectively. Let $\nabla$ denote the covariant differentiation on $M^{n}$. By writing $R_{i j, k}=\nabla_{k} R_{i j}$ and $R_{i j, k l}=\nabla_{l} \nabla_{k} R_{i j}$, etc., we have the Ricci formulas

$$
\begin{gathered}
R_{i j, k l}-R_{i j, l k}=\sum_{t} R_{t j} R_{t i k l}+\sum_{t} R_{i t} R_{t j k l} \\
R_{i j, k l m}-R_{i j, k m l}=\sum_{t} R_{t j, k} R_{t i l m}+\sum_{t} R_{i t, k} R_{t j l m}+\sum_{t} R_{i j, t} R_{t k l m} .
\end{gathered}
$$

The Bek tensor with the components $C_{i j k}$ is defined by

$$
C_{i j k}=\frac{1}{n-2}\left(R_{i j, k}-R_{i k, j}\right)-\frac{1}{2(n-1)(n-2)}\left(\delta_{i j} r_{k}-\delta_{i k} r_{j}\right)
$$

where $r_{k}=\nabla_{k} r$. 
For $n \geq 4$, we know that $M^{n}$ is a locally conformally flat Riemannian manifold if and only if $C_{i j k l}=0$, and that $C_{i j k}=0$ on $M^{n}$ in this case.

When $n=3$, we always have $C_{i j k l}=0$ on $M^{3}$ and that $M^{3}$ is a locally conformally flat Riemannian manifold if and only if $C_{i j k}=0$. Hence, if $M^{n}$ is a locally conformally flat $n$-dimensional Riemannian manifold, then

$$
\begin{aligned}
R_{i j k l}= & \frac{1}{n-2}\left(R_{i k} \delta_{j l}-R_{i l} \delta_{j k}+R_{j l} \delta_{i k}-R_{j k} \delta_{i l}\right) \\
& -\frac{r}{(n-1)(n-2)}\left(\delta_{i k} \delta_{j l}-\delta_{i l} \delta_{j k}\right) .
\end{aligned}
$$

Moreover if the scalar curvature $r$ is constant, then we obtain

$$
R_{i j, k}=R_{i k, j}
$$

From now on, we assume $n=3$. Let $\lambda_{i}$ for $i=1,2,3$ be the eigenvalues of Ricci curvature tensor $\left(R_{i j}\right)$ of $M^{3}$ and define $\mu_{i}:=\lambda_{i}-\frac{r}{3}$. We put $B=S-\frac{r^{2}}{3}$. The following has been proved in [1]:

Proposition. Let $M^{3}$ be a locally conformally flat Riemannian manifold of dimension 3. If the scalar curvature and the squared norm of Ricci curvature tensor of $M^{3}$ are constant, then we have

$$
\begin{aligned}
\sum_{i, j, k} R_{i j, k}^{2} & =-3 \sum_{i} \mu_{i}^{3}-\frac{r}{2} B \\
\sum_{i, j, k, l} R_{i j, k l}^{2} & =-24 \sum_{i, j, k} \mu_{i} R_{i j, k}^{2}-\frac{3}{4} r \sum_{i, j, k} R_{i j, k}^{2}-\frac{9}{4} B\left(B-\frac{r^{2}}{6}\right) .
\end{aligned}
$$

In order to prove the Main Theorem, the Theorem 3 in [1] will play an important role. We restate it in the following:

Theorem 3 (see [1]). Let $M^{3}$ be a complete locally conformally flat 3-dimensional Riemannian manifold with constant scalar curvature $r$ and constant squared norm $S$ of the Ricci curvature tensor. If $r<0$, then we have

$$
\sup \sum_{i=1}^{3} \mu_{i}^{3}=\frac{B^{\frac{3}{2}}}{\sqrt{6}}
$$

\section{Proof of Main Theorem}

In order to prove Main Theorem, first, we discuss the following special case: 
Theorem 3.0. There exist no locally conformally flat 3-dimensional Riemannian manifolds with the negative constant scalar curvature $r$ and Ricci curvature tensor of constant squared norm $S$ satisfying $\frac{r^{2}}{3}+\left(\frac{7}{113}\right)^{2} \frac{2 r^{2}}{3}<S<\frac{r^{2}}{3}+$ $\left(\frac{35}{137}\right)^{2} \frac{r^{2}}{6}$ such that $\sum_{i}\left(\lambda_{i}-\frac{r}{3}\right)^{3}=\frac{\left(S-\frac{r^{2}}{3}\right)^{\frac{3}{2}}}{\sqrt{6}}$ holds at some point $p$.

From the Lemma 1 in [1], we know that

$$
-\frac{B^{\frac{3}{2}}}{\sqrt{6}} \leq \sum_{i} \mu_{i}^{3} \leq \frac{B^{\frac{3}{2}}}{\sqrt{6}},
$$

where the equality holds if and only if at least two of $\mu_{i}$ are equal. From the assumption of Theorem 3.0, we have, at a point $p, \sum_{i} \mu_{i}^{3}=\frac{B^{\frac{3}{2}}}{\sqrt{6}}$. Hence, for the purpose of proving this Theorem 3.0 , we can assume that at the point $p$

$$
\lambda_{1}=\lambda_{2}=\lambda \quad \text { and } \quad \lambda_{3}=r-2 \lambda
$$

that is,

$$
\mu_{1}=\mu_{2}=\mu \quad \text { and } \quad \mu_{3}=-2 \mu \text {. }
$$

If $\mu=0$ at the point $p$, then $\mu_{1}=\mu_{2}=\mu_{3}=0$ at $p$. Hence, $S-\frac{r^{2}}{3}=B=$ $\sum_{i} \mu_{i}^{2}=0$ at $p$. Since $S>\frac{r^{2}}{3}+\left(\frac{7}{113}\right)^{2} \frac{2 r^{2}}{3}$, that is, $B>\left(\frac{7}{113}\right)^{2} \frac{2 r^{2}}{3}$, we can assume $\mu \neq 0$ at the point $p$. Then we obtain from (3.2) at $p$

$$
B=6 \mu^{2} .
$$

Let $\left\{e_{1}, e_{2}, e_{3}\right\}$ be an orthonormal basis of tangent space $T_{p} M$ at $p$. Thus we have at $p$

$$
\left(R_{i j}\right)=\left(\begin{array}{ccc}
\lambda & 0 & 0 \\
0 & \lambda & 0 \\
0 & 0 & r-2 \lambda
\end{array}\right)
$$

and

$$
\left(\tau_{i j}\right)=\left(\begin{array}{ccc}
\mu & 0 & 0 \\
0 & \mu & 0 \\
0 & 0 & -2 \mu
\end{array}\right),
$$

where $\tau_{i j}=R_{i j}-\frac{r}{3} \delta_{i j}$. Because $\left\{e_{1}, e_{2}\right\}$ is a basis of the eigenspace corresponding to the $\lambda$-eigenvalue of $\left(R_{i j}\right)$ at $p$, we can assume $R_{12,3}=0$ at $p$, rotating it if necessary.

Lemma 3.1. At the point $p$, we have

$$
R_{22, k}=-R_{11, k}, \quad R_{33, k}=0 \quad \text { for } k=1,2,3,
$$




$$
\sum_{i, j, k} R_{i j, k}^{2}=6 R_{11,3}^{2}+4\left(R_{11,1}^{2}+R_{22,2}^{2}\right)=3 B\left(\mu-\frac{r}{6}\right) .
$$

Proof. By differentiating $\sum_{i} R_{i i}=r$ and $\sum_{i, j} R_{i j}^{2}=S$ at $p$, we have respectively

$$
\sum_{i} R_{i i, k}=0 \quad \text { and } \quad \sum_{i} R_{i i} R_{i i, k}=0 .
$$

Hence, at $p$, we have $R_{22, k}=-R_{11, k}$ and $R_{33, k}=0$ for $k=1,2,3$. Therefore, by using (2.3), we obtain also at $p$

$$
\sum_{i, j, k} R_{i j, k}^{2}=6 R_{11,3}^{2}+4\left(R_{11,1}^{2}+R_{22,2}^{2}\right)=3 B\left(\mu-\frac{r}{6}\right) .
$$

This completes the proof of Lemma 3.1.

Let us denote $A_{1}$ and $A_{2}$ by

$$
A_{1}=R_{11,3}^{2} \quad \text { and } \quad A_{2}=R_{11,1}^{2}+R_{22,2}^{2} .
$$

Then (3.5) in Lemma 3.1 means

$$
6 A_{1}+4 A_{2}=3 B\left(\mu-\frac{r}{6}\right)
$$

Lemma 3.2. If $\{i, j, k, l\}$ is not a permutation of $\{1,1,3,3\}$ or $\{2,2,3,3\}$, then, at $p$,

$$
R_{i j, k l}=R_{i j, l k}
$$

Proof. From the Ricci formulas and (2.1), we obtain at $p$

$$
R_{i j, k l}-R_{i j, l k}=\left(\mu_{i}-\mu_{j}\right)\left(\mu_{i}+\mu_{j}+\frac{r}{6}\right)\left(\delta_{i k} \delta_{j l}-\delta_{i l} \delta_{j k}\right) .
$$

If $\{i, j, k, l\}$ is not a permutation of $\{1,1,3,3\}$ or $\{2,2,3,3\}$, we have at $p$

$$
R_{i j, k l}=R_{i j, l k} \text {. }
$$

Thus, we have finished the proof of Lemma 3.2.

Lemma 3.3. At the point $p$,

$$
\begin{aligned}
& R_{11,11}=R_{22,22}, \quad R_{33,33}=\frac{2 A_{1}}{3 \mu}, \quad R_{33,12}=0, \quad R_{33,13}=\frac{2 R_{11,1} R_{11,3}}{3 \mu}, \\
& R_{33,23}=-\frac{2 R_{22,2} R_{11,3}}{3 \mu}, \quad R_{33,11}=R_{33,22}=\frac{2\left(A_{1}+A_{2}\right)}{3 \mu}, \\
& R_{11,33}=R_{22,33}=-\frac{A_{1}}{3 \mu} .
\end{aligned}
$$


Proof. Since $\sum_{i} R_{i i}=r$ and $\sum_{i, j} R_{i j}^{2}=S$ are constants, we have, by differentiating them at $p$,

$$
\sum_{i} R_{i i, k l}=0
$$

and

$$
\sum_{i} \mu_{i} R_{i i, k l}+\sum_{i, j} R_{i j, k} R_{i j, l}=0 .
$$

Hence, by direct calculation it follows that

$$
R_{33, k l}=\frac{1}{3 \mu} \sum_{i, j} R_{i j, k} R_{i j, l} .
$$

That is, by using (3.7), it follows that

$$
\begin{aligned}
& R_{33,12}=0, \quad R_{33,33}=\frac{1}{3 \mu} \sum_{i, j} R_{i j, 3}^{2}=\frac{2 A_{1}}{3 \mu} \\
& R_{33,13}=\frac{1}{3 \mu} \sum_{i, j} R_{i j, 1} R_{i j, 3}=\frac{2 R_{11,1} R_{11,3}}{3 \mu} \\
& R_{33,23}=\frac{1}{3 \mu} \sum_{i, j} R_{i j, 2} R_{i j, 3}=-\frac{2 R_{22,2} R_{11,3}}{3 \mu}, \\
& R_{33,11}=R_{33,22}=\frac{1}{3 \mu} \sum_{i, j} R_{i j, 1}^{2}=\frac{2\left(A_{1}+A_{2}\right)}{3 \mu}, \\
& R_{11,33}=R_{22,33}=R_{33,11}+\left(\mu_{1}-\mu_{3}\right)\left(\mu_{1}+\mu_{3}+\frac{r}{6}\right)=-\frac{A_{1}}{3 \mu}
\end{aligned}
$$

at the point $p$. According to (3.8), we have also at $p$

$$
R_{11,11}=R_{22,22} \text {. }
$$

This completes the proof of Lemma 3.3.

Lemma 3.4. At the point p, we have

$$
\begin{aligned}
\mu^{2}\{ & \left.2\left(R_{11,11}^{2}+R_{11,12}^{2}\right)+3\left(R_{11,23}^{2}+R_{22,13}^{2}\right)+R_{11,13}^{2}+R_{22,23}^{2}\right\} \\
+ & 2\left(A_{1}+A_{2}\right) \mu R_{11,11} \\
& =-\frac{58 A_{1}^{2}+112 A_{1} A_{2}+48 A_{2}^{2}}{36}-4 \mu B A_{2}+\frac{3 \mu^{2}}{16}\left(r^{2}-3 r \mu-3 B\right) B .
\end{aligned}
$$


Proof. By calculating $\sum_{i, j, k} R_{i j, k 1}^{2}, \sum_{i, j, k} R_{i j, k 2}^{2}$ and $\sum_{i, j, k} R_{i j, k 3}^{2}$ separately, we have at $p$

$$
\begin{aligned}
\sum_{i, j, k} R_{i j, k 1}^{2}= & 6 R_{12,31}^{2}+\sum_{i} R_{i i, i 1}^{2}+3 \sum_{i \neq k} R_{i i, k 1}^{2} \\
= & 3\left(R_{11,21}^{2}+R_{11,31}^{2}+R_{22,11}^{2}+R_{22,31}^{2}+R_{33,11}^{2}+R_{33,21}^{2}\right) \\
& +6 R_{11,23}^{2}+R_{11,11}^{2}+R_{22,21}^{2}+R_{33,31}^{2} \\
= & 3\left(2 R_{11,23}^{2}+R_{11,13}^{2}+R_{22,13}^{2}\right)+R_{33,31}^{2} \\
& +6\left(R_{11,11} R_{33,11}+R_{33,11}^{2}\right)+4\left(R_{11,12}^{2}+R_{11,11}^{2}\right)
\end{aligned}
$$

$$
\begin{aligned}
\sum_{i, j, k} R_{i j, k 2}^{2}= & 6 R_{12,32}^{2}+\sum_{i} R_{i i, i 2}^{2}+3 \sum_{i \neq k} R_{i i, k 2}^{2} \\
= & 3\left(R_{11,22}^{2}+R_{11,32}^{2}+R_{22,12}^{2}+R_{22,32}^{2}+R_{33,12}^{2}+R_{33,22}^{2}\right) \\
& +6 R_{22,13}^{2}+R_{11,12}^{2}+R_{22,22}^{2}+R_{33,32}^{2} \\
= & 3\left(2 R_{11,11} R_{33,11}+R_{33,11}^{2}+R_{33,22}^{2}\right)+4\left(R_{11,12}^{2}+R_{11,11}^{2}\right) \\
& +3\left(2 R_{22,13}^{2}+R_{11,23}^{2}+R_{22,23}^{2}\right)+R_{33,23}^{2} \\
\sum_{i, j, k} R_{i j, k 3}^{2}=6 & R_{12,33}^{2}+\sum_{i} R_{i i, i 3}^{2}+3 \sum_{i \neq k} R_{i i, k 3}^{2} \\
= & 3\left(R_{11,23}^{2}+R_{11,33}^{2}+R_{22,13}^{2}+R_{22,33}^{2}+R_{33,13}^{2}+R_{33,23}^{2}\right) \\
& +R_{11,13}^{2}+R_{22,23}^{2}+R_{33,33}^{2} \\
= & 3\left(R_{11,23}^{2}+R_{22,13}^{2}\right)+\left(R_{11,13}^{2}+R_{22,23}^{2}\right)+\left(\frac{2 A_{1}}{3 \mu}\right)^{2} \\
& +3\left\{2\left(\frac{-A_{1}}{3 \mu}\right)^{2}+\left(\frac{2 R_{11,1} R_{11,3}}{3 \mu}\right)^{2}+\left(\frac{-2 R_{22,2} R_{11,3}}{3 \mu}\right)^{2}\right\} .
\end{aligned}
$$

Here, we used the relations $R_{11,11}+R_{22,11}+R_{33,11}=0, R_{33,21}=0, R_{22,21}=-R_{11,21}$ and so on in (3.8) and Lemma 3.3. Therefore, by summing up these equations (3.9), (3.10) and (3.11), we obtain at $p$

$$
\begin{aligned}
\sum_{i, j, k, l} R_{i j, k l}^{2}= & 12\left(R_{11,23}^{2}+R_{22,13}^{2}\right)+4\left(R_{11,13}^{2}+R_{22,23}^{2}\right) \\
& +8\left(R_{11,12}^{2}+R_{11,11}^{2}\right)+\frac{10 A_{1}^{2}+12 A_{1} A_{2}}{9 \mu^{2}} \\
& +\frac{16\left(A_{1}+A_{2}\right)^{2}}{3 \mu^{2}}+\frac{4 A_{1} A_{2}}{9 \mu^{2}}+\frac{8\left(A_{1}+A_{2}\right)}{\mu} R_{11,11}
\end{aligned}
$$


The other hand, by using (2.4), we have at $p$

$$
\begin{aligned}
\sum_{i, j, k, l} R_{i j, k l}^{2} & =-24\left(4 \mu A_{2}\right)-\frac{3 r}{4} 3 B\left(\mu-\frac{r}{6}\right)-\frac{9}{4} B\left(B-\frac{r^{2}}{6}\right) \\
& =-96 \mu A_{2}+\frac{3 B}{4}\left(r^{2}-3 r \mu-3 B\right) .
\end{aligned}
$$

Hence, from (3.12) and (3.13), we obtain at $p$

$$
\begin{aligned}
\mu^{2}\{ & \left.2\left(R_{11,11}^{2}+R_{11,12}^{2}\right)+3\left(R_{11,23}^{2}+R_{22,13}^{2}\right)+R_{11,13}^{2}+R_{22,23}^{2}\right\} \\
+ & 2\left(A_{1}+A_{2}\right) \mu R_{11,11} \\
& =-\frac{58 A_{1}^{2}+112 A_{1} A_{2}+48 A_{2}^{2}}{36}-4 \mu B A_{2}+\frac{3 \mu^{2}}{16}\left(r^{2}-3 r \mu-3 B\right) B .
\end{aligned}
$$

This proves Lemma 3.4.

Lemma 3.5. At the point $p$, it follows that

$$
\begin{aligned}
\sum_{i, j, k} R_{i j, k} R_{i j, k 33}= & -3\left(R_{11,23}^{2}+R_{22,13}^{2}\right)-\left(R_{11,13}^{2}+R_{22,23}^{2}\right) \\
& -\frac{9}{2}\left(2 \sum_{i, j} \mu_{i} R_{i j, 3}^{2}+\sum_{i} \mu_{i}^{2} R_{i i, 33}\right)-\frac{10 A_{1}^{2}+12 A_{1} A_{2}}{9 \mu^{2}} .
\end{aligned}
$$

Proof. By differentiating at $p$ the both sides of

$$
\sum_{i, j, k} R_{i j, k}^{2}=-3 \sum_{i} \mu_{i}^{3}-\frac{r}{2} B
$$

obtained in Proposition, we have

$$
2 \sum_{i, j, k} R_{i j, k} R_{i j, k 33}+2 \sum_{i, j, k} R_{i j, k 3}^{2}=-3 \nabla_{3} \nabla_{3} \sum_{i} \mu_{i}^{3}
$$

and we obtain at $p$, by directly calculating,

$$
\nabla_{3} \nabla_{3} \sum_{i} \mu_{i}^{3}=6 \sum_{i, j} \mu_{i} R_{i j, 3}^{2}+3 \sum_{i} \mu_{i}^{2} R_{i i, 33} .
$$

Thus, from (3.11), (3.14) and (3.15), we conclude

$$
\begin{aligned}
\sum_{i, j, k} R_{i j, k} R_{i j, k 33}= & -3\left(R_{11,23}^{2}+R_{22,13}^{2}\right)-\left(R_{11,13}^{2}+R_{22,23}^{2}\right) \\
& -\frac{9}{2}\left(2 \sum_{i, j} \mu_{i} R_{i j, 3}^{2}+\sum_{i, j} \mu_{i}^{2} R_{i i, 33}\right)-\frac{10 A_{1}^{2}+12 A_{1} A_{2}}{9 \mu^{2}},
\end{aligned}
$$


that is, the proposed relation in Lemma 3.5 holds.

Lemma 3.6. At the point p, we have

$$
\sum_{i, j, k} R_{i j, k} R_{33, i j k}=\frac{4}{3 \mu^{2}}\left(2 A_{1}^{2}-A_{2}^{2}\right)+\frac{4 A_{1}}{\mu} R_{11,11} .
$$

Proof. By differentiating $\sum_{i} R_{i i}=r$ and $\sum_{i, j} R_{i j}^{2}=S$ at $p$, we have,

$$
\sum_{i} R_{i i, k l m}=0
$$

and

$$
\sum_{i, j} R_{i j} R_{i j, k l m}+\sum_{i, j} R_{i j, m} R_{i j, k l}+\sum_{i, j} R_{i j, k} R_{i j, l m}+\sum_{i, j} R_{i j, l} R_{i j, k m}=0
$$

respectively. Therefore, from (3.16), we obtain at $p$

$$
\sum_{i, j} R_{i j} R_{i j, k l m}=-3 \mu R_{33, k l m}
$$

Hence, it follows that at $p$

$$
\begin{aligned}
\mu & \sum_{k, l, m} R_{k l, m} R_{33, k l m} \\
= & \sum_{i, j, k, l, m} R_{k l, m} R_{i j, m} R_{i j, k l} \\
= & \sum_{k, l, m} R_{k l, m} R_{11, k}\left(R_{11, m l}-R_{22, m l}\right)+2 \sum_{k, l, m} R_{k l, m} R_{12, k} R_{12, m l} \\
& \quad+2 \sum_{k, l, m} R_{k l, m} R_{13, k} R_{13, m l}+2 \sum_{k, l, m} R_{k l, m} R_{23, k} R_{23, m l} .
\end{aligned}
$$

Now, by calculating the terms in (3.18) separately, we have at $p$ respectively

$$
\begin{aligned}
& \sum_{k, l, m} R_{k l, m} R_{11, k}\left(R_{11, m l}-R_{22, m l}\right) \\
& =2\left(A_{1}+A_{2}\right)\left(R_{11,11}-R_{22,11}\right)+2 R_{11,1} R_{11,3}\left(R_{11,13}-R_{22,13}\right) \\
& \quad+2 R_{22,2} R_{11,3}\left(R_{11,23}-R_{22,23}\right) \\
& \quad 2 \sum_{k, l, m} R_{k l, m} R_{12, k} R_{12, m l} \\
& \quad=4 A_{2} R_{11,22}-4 R_{22,2} R_{11,3} R_{11,23}+4 R_{11,1} R_{11,3} R_{22,13},
\end{aligned}
$$




$$
\begin{aligned}
2 \sum_{k, l, m} & R_{k l, m} R_{13, k} R_{13, m l} \\
= & 2 A_{1}\left(R_{11,33}+R_{33,11}\right)+2 R_{11,1} R_{11,3} R_{11,13} \\
& -2 R_{11,1} R_{11,3} R_{22,13}-4 R_{22,2} R_{11,3} R_{11,23},
\end{aligned}
$$

$$
\begin{aligned}
2 \sum_{k, l, m} & R_{k l, m} R_{23, k} R_{23, m l} \\
= & 2 A_{1}\left(R_{22,33}+R_{33,22}\right)+2 R_{22,2} R_{11,3}\left(R_{11,23}-R_{22,23}\right) \\
& +4 R_{11,1} R_{11,3} R_{22,13} .
\end{aligned}
$$

From (3.18), (3.19), (3.20), (3.21) and (3.22), we obtain at $p$, by making use of the relation $R_{11,11}+R_{22.11}+R_{33,11}=0$,

$$
\mu \sum_{k l, m} R_{k l, m} R_{33, k l m}=\frac{4}{3 \mu}\left(2 A_{1}^{2}-A_{2}^{2}\right)+4 A_{1} R_{11,11}
$$

That is, Lemma 3.6 holds.

LEMma 3.7. At the point p, we obtain

$$
\begin{aligned}
\mu^{2}\left\{3\left(R_{11,23}^{2}+R_{22,13}^{2}\right)+\left(R_{11,13}^{2}+R_{22,23}^{2}\right)\right\} \\
=-\frac{10 A_{1}^{2}+12 A_{1} A_{2}}{9}-27 A_{1} \mu^{3}-\frac{4}{3}\left(2 A_{1}^{2}-A_{2}^{2}\right) \\
\quad-3 r \mu^{2} A_{1}-\left(4 \mu+\frac{4}{3} r\right) \mu^{2} A_{2}-4 A_{1} \mu R_{11,11} .
\end{aligned}
$$

Proof. We write

$$
R_{i j k l, m}=\nabla_{m} R_{i j k l}
$$

Then, since

$$
\begin{aligned}
R_{33, i j k}-R_{i j, k 33} & \\
= & \nabla_{k}\left(R_{i j, 33}+\sum_{m} R_{m i} R_{m 33 j}+\sum_{m} R_{3 m} R_{m i 3 j}\right)-R_{i j, k 33} \\
= & R_{i j, 33 k}+\sum_{m} R_{m i, k} R_{m 33 j}+\sum_{m} R_{m i} R_{m 33 j, k} \\
& \quad+\sum_{m} R_{3 m, k} R_{m i 3 j}+\sum_{m} R_{3 m} R_{m i 3 j, k}-R_{i j, k 33} \\
= & R_{i j, 3 k 3}+\sum_{m} R_{m j, 3} R_{m i 3 k}+\sum_{m} R_{i m, 3} R_{m j 3 k}
\end{aligned}
$$




$$
\begin{aligned}
& +\sum_{m} R_{i j, m} R_{m 33 k}+\sum_{m} R_{m i, k} R_{m 33 j}+\sum_{m} R_{m i} R_{m 33 j, k} \\
& +\sum_{m} R_{3 m, k} R_{m i 3 j}+\sum_{m} R_{3 m} R_{m i 3 j, k}-R_{i j, k 33} \\
= & \nabla_{3}\left(R_{i j, k 3}+\sum_{m} R_{m j} R_{m i 3 k}+\sum_{m} R_{i m} R_{m j 3 k}\right)+\sum_{m} R_{m j, 3} R_{m i 3 k} \\
& +\sum_{m} R_{i m, 3} R_{m j 3 k}+\sum_{m} R_{i j, m} R_{m 33 k}+\sum_{m} R_{m i, k} R_{m 33 j} \\
& +\sum_{m} R_{m i} R_{m 33 j, k}+\sum_{m} R_{3 m, k} R_{m i 3 j}+\sum_{m} R_{3 m} R_{m i 3 j, k}-R_{i j, k 33} \\
= & \sum_{m} R_{m j, 3} R_{m i 3 k}+\sum_{m} R_{i m, 3} R_{m j 3 k}+\sum_{m} R_{m j} R_{m i 3 k, 3} \\
& +\sum_{m} R_{i m} R_{m j 3 k, 3}+\sum_{m} R_{m j, 3} R_{m i 3 k}+\sum_{m} R_{i m, 3} R_{m j 3 k} \\
& +\sum_{m} R_{i j, m} R_{m 33 k}+\sum_{m} R_{m i, k} R_{m 33 j}+\sum_{m} R_{m i} R_{m 33 j, k} \\
& +\sum_{m} R_{3 m, k} R_{m i 3 j}+\sum_{m} R_{3 m} R_{m i 3 j, k},
\end{aligned}
$$

we obtain at $p$

$$
\begin{aligned}
& \sum_{i, j, k} R_{i j, k}\left(R_{33, i j k}-R_{i j, k 33}\right) \\
&=\sum_{i, j, k, m} R_{i j, k}\left\{5 R_{m j, 3} R_{m i 3 k}+2 R_{i j, m} R_{m 33 k}\right. \\
&\left.\quad+\left(\lambda_{i}-\lambda_{j}\right) R_{i j 3 k, 3}+\left(\lambda_{i}-\lambda_{3}\right) R_{i 33 j, k}\right\} .
\end{aligned}
$$

Since $M^{3}$ is a 3 -dimensional Riemannian manifold, we have

$$
R_{i j k l}=\left(R_{i k} \delta_{j l}-R_{i l} \delta_{j k}+\delta_{i k} R_{j l}-\delta_{i l} R_{j k}\right)-\frac{r}{2}\left(\delta_{i k} \delta_{j l}-\delta_{i l} \delta_{j k}\right) .
$$

So, we obtain

$$
R_{i j k l, m}=R_{i k, m} \delta_{j l}-R_{i l, m} \delta_{j k}+\delta_{i k} R_{j l, m}-\delta_{i l} R_{j k, m} .
$$

Because $R_{i j}=\lambda_{i} \delta_{i j}$ at the point $p$, by the direct calculations, we also have the following relations

$$
R_{i j k l}=\left(\lambda_{i}+\lambda_{j}-\frac{r}{2}\right)\left(\delta_{i k} \delta_{j l}-\delta_{i l} \delta_{j k}\right)
$$


$(3.29)$

$$
\begin{aligned}
& \sum_{i, j, k, m} R_{i j, k} R_{m j, 3} R_{m i 3 k} \\
& =\sum_{i, j, k, m} R_{i j, k} R_{m j, 3}\left(\lambda_{m}+\lambda_{i}-\frac{r}{2}\right)\left(\delta_{m 3} \delta_{i k}-\delta_{m k} \delta_{i 3}\right) \\
& \quad=-\sum_{i, j} R_{i j, 3}^{2}\left(\lambda_{i}+\lambda_{3}-\frac{r}{2}\right)
\end{aligned}
$$

$$
\begin{aligned}
& \sum_{i, j, k, m} R_{i j, k} R_{i j, m} R_{m 33 k} \\
& =\sum_{i, j, k, m} R_{i j, k} R_{i j, m}\left(\lambda_{m}+\lambda_{3}-\frac{r}{2}\right)\left(\delta_{m 3} \delta_{3 k}-\delta_{m k} \delta_{33}\right) \\
& =\sum_{i, j} R_{i j, 3}^{2}\left(2 \lambda_{3}-\frac{r}{2}\right)-\sum_{i, j, k} \lambda_{i} R_{i j, k}^{2}+\frac{r}{2} \sum_{i, j, k} R_{i j, k}^{2}-\lambda_{3} \sum_{i, j, k} R_{i j, k}^{2}
\end{aligned}
$$

$$
\begin{aligned}
\sum_{i, j, k} & R_{i j, k}\left(\lambda_{i}-\lambda_{j}\right) R_{i j 3 k, 3} \\
& =\sum_{i, j, k} R_{i j, k}\left(\lambda_{j}-\lambda_{i}\right)\left(R_{i k, 3} \delta_{j 3}-R_{i 3,3} \delta_{j k}+\delta_{i k} R_{j 3,3}-\delta_{i 3} R_{j k, 3}\right) \\
& =2 \lambda_{3} \sum_{i, j} R_{i j, 3}^{2}-2 \sum_{i, j} \lambda_{i} R_{i j, 3}^{2}
\end{aligned}
$$

$$
\begin{aligned}
\sum_{i, j, k} & R_{i j, k}\left(\lambda_{i}-\lambda_{3}\right) R_{i 33 j, k} \\
& =\sum_{i, j, k} R_{i j, k}\left(\lambda_{i}-\lambda_{3}\right)\left(R_{i 3, k} \delta_{3 j}-R_{i j, k} \delta_{33}+\delta_{i 3} R_{3 j, k}-\delta_{i j} R_{33, k}\right) \\
& =\sum_{i, j} \lambda_{i} R_{i j, 3}^{2}-\lambda_{3} \sum_{i, j} R_{i j, 3}^{2}-\sum_{i, j, k} \lambda_{i} R_{i j, k}^{2}+\lambda_{3} \sum_{i, j, k} R_{i j, k}^{2} .
\end{aligned}
$$

Therefore, by summing up (3.25), (3.29), (3.30), (3.31) and (3.32), we have at $p$,

$$
\begin{aligned}
\sum_{i, j, k} & R_{i j, k}\left(R_{33, i j k}-R_{i j, k 33}\right) \\
= & -5 \sum_{i, j} R_{i j, 3}^{2}\left(\lambda_{i}+\lambda_{3}-\frac{r}{2}\right)+2 \sum_{i, j} R_{i j, 3}^{2}\left(2 \lambda_{3}-\frac{r}{2}\right)-2 \sum_{i, j, k} \lambda_{i} R_{i j, k}^{2} \\
& +r \sum_{i, j, k} R_{i j, k}^{2}-2 \lambda_{3} \sum_{i, j, k} R_{i j, k}^{2}+2 \lambda_{3} \sum_{i, j} R_{i j, 3}^{2}-2 \sum_{i, j} \lambda_{i} R_{i j, 3}^{2} \\
& +\sum_{i, j} \lambda_{i} R_{i j, 3}^{2}-\lambda_{3} \sum_{i, j} R_{i j, 3}^{2}-\sum_{i, j, k} \lambda_{i} R_{i j, k}^{2}+\lambda_{3} \sum_{i, j, k} R_{i j, k}^{2}
\end{aligned}
$$


400

$$
\begin{aligned}
= & -12\left(\mu+\frac{r}{3}\right) A_{1}+3 r A_{1}-3\left\{4 \mu A_{2}+\frac{r}{3}\left(6 A_{1}+4 A_{2}\right)\right\} \\
& +\left(2 \mu-\frac{r}{3}\right)\left(6 A_{1}+4 A_{2}\right)+r\left(6 A_{1}+4 A_{2}\right) \\
= & -3 r A_{1}-\left(4 \mu+\frac{4}{3} r\right) A_{2} .
\end{aligned}
$$

Here, we used the following relations at $p$

$$
\begin{aligned}
\sum_{i, j} R_{i j, 3}^{2} & =2 R_{11,3}^{2}=2 A_{1}, \\
\sum_{i, j, k} R_{i j, k}^{2} & =6 A_{1}+4 A_{2}, \\
\sum_{i, j, k} \lambda_{i} R_{i j, k}^{2} & =\sum_{i, j, k} \mu_{i} R_{i j, k}^{2}+\frac{r}{3} \sum_{i, j, k} R_{i j, k}^{2} \\
& =4 \mu A_{2}+\frac{r}{3}\left(6 A_{1}+4 A_{2}\right) \\
\sum_{i, j} \lambda_{i} R_{i j, 3}^{2} & =2\left(\mu+\frac{r}{3}\right) A_{1} .
\end{aligned}
$$

Therefore, from Lemma 3.5, Lemma 3.6 and (3.33), we conclude

$$
\begin{aligned}
& 3\left(R_{11,23}^{2}+R_{22,13}^{2}\right)+\left(R_{11,13}^{2}+R_{22,23}^{2}\right)+\frac{10 A_{1}^{2}+12 A_{1} A_{2}}{9 \mu^{2}} \\
& +9 \sum_{i, j} \mu_{i} R_{i j, 3}^{2}+\frac{9}{2} \sum_{i, j} \mu_{i}^{2} R_{i i, 33}+\frac{4}{3 \mu^{2}}\left(2 A_{1}^{2}-A_{2}^{2}\right)+\frac{4 A_{1}}{\mu} R_{11,11} \\
& \quad=-3 r A_{1}-\left(4 \mu+\frac{4}{3} r\right) A_{2} .
\end{aligned}
$$

Since we have at $p$ by Lemma 3.3

$$
\begin{aligned}
\sum_{i} \mu_{i}^{2} R_{i i, 33} & =\mu^{2}\left(R_{11,33}+R_{22,33}\right)+4 \mu^{2} R_{33,33} \\
& =2 \mu A_{1}
\end{aligned}
$$

it folows from (3.38) that

$$
\begin{aligned}
\mu^{2}\left\{3\left(R_{11,23}^{2}+R_{22,13}^{2}\right)+\left(R_{11,13}^{2}+R_{22,23}^{2}\right)\right\} \\
=-\frac{10 A_{1}^{2}+12 A_{1} A_{2}}{9}-27 A_{1} \mu^{3}-\frac{4}{3}\left(2 A_{1}^{2}-A_{2}^{2}\right) \\
\quad-3 r \mu^{2} A_{1}-\left(4 \mu+\frac{4}{3} r\right) \mu^{2} A_{2}-4 \mu A_{1} R_{11,11},
\end{aligned}
$$


that is, Lemma 3.7 holds.

LEMMA 3.8. At the point p, we have

$$
\begin{aligned}
& 2 \mu^{2}\left(R_{11,11}^{2}+R_{11,12}^{2}\right)+2\left(A_{2}-A_{1}\right) \mu R_{11,11} \\
& =\frac{39 A_{1}^{2}-32 A_{1} A_{2}-48 A_{2}^{2}}{18}+\left(\frac{9}{2} B \mu+3 r \mu^{2}\right) A_{1} \\
& \quad+\left(\frac{2}{9} r-\frac{10 \mu}{3}\right) B A_{2}-\left(\frac{3}{8} r+\frac{9}{8} \mu\right) \mu^{2}\left(6 A_{1}+4 A_{2}\right) .
\end{aligned}
$$

Proof. According to (3.7), we have at $p$

$$
\begin{aligned}
\frac{3 B \mu^{2}}{16}\left(r^{2}-3 r \mu-3 B\right) & =-\left(\frac{9}{8} \mu+\frac{3}{8} r\right) \mu^{2}\left\{3 B\left(\mu-\frac{r}{6}\right)\right\} \\
& =-\left(\frac{3}{8} r+\frac{9}{8} \mu\right) \mu^{2}\left(6 A_{1}+4 A_{2}\right) .
\end{aligned}
$$

From Lemma 3.4 and Lemma 3.7, we also obtain at $p$

$$
\begin{aligned}
2 \mu^{2}( & \left.R_{11,11}^{2}+R_{11,12}^{2}\right)+2\left(A_{2}+A_{1}\right) \mu R_{11,11}-4 A_{1} \mu R_{11,11} \\
= & \frac{10 A_{1}^{2}+12 A_{1} A_{2}}{9}+27 A_{1} \mu^{3}+\frac{4}{3}\left(2 A_{1}^{2}-A_{2}^{2}\right)+3 r \mu^{2} A_{1} \\
& +\left(4 \mu+\frac{4}{3} r\right) \mu^{2} A_{2}-\frac{58 A_{1}^{2}+112 A_{1} A_{2}+48 A_{2}^{2}}{36} \\
& \quad-4 B \mu A_{2}+\frac{3 B \mu^{2}}{16}\left(r^{2}-3 r \mu-3 B\right) .
\end{aligned}
$$

Together with (3.41), we get at $p$

$$
\begin{aligned}
2 \mu^{2}( & \left.R_{11,11}^{2}+R_{11,12}^{2}\right)+2\left(A_{2}-A_{1}\right) \mu R_{11,11} \\
= & \frac{39 A_{1}^{2}-32 A_{1} A_{2}-48 A_{2}^{2}}{18}+\left(\frac{9}{2} B \mu+3 r \mu^{2}\right) A_{1} \\
& \quad+\left(\frac{2}{9} r-\frac{10 \mu}{3}\right) B A_{2}-\left(\frac{3}{8} r+\frac{9}{8} \mu\right) \mu^{2}\left(6 A_{1}+4 A_{2}\right) .
\end{aligned}
$$

This completes the proof of Lemma 3.8.

Proof of Theorem 3.0. We suppose that there exists a complete locally conformally flat 3-dimensional Riemannian manifold which satisfies the assumptions in Theorem 3.0. According to the Lemma 1 in [1], we know that, at each point in $M^{3}$,

$$
-\frac{B^{\frac{3}{2}}}{\sqrt{6}} \leq \sum_{i} \mu_{i}^{3} \leq \frac{B^{\frac{3}{2}}}{\sqrt{6}}
$$


where the equality holds if and only if at least two of $\mu_{i}$ are equal. From the assumptions of Theorem 3.0, we have at a point $p$,

$$
\sum_{i} \mu_{i}^{3}=\frac{B^{\frac{3}{2}}}{\sqrt{6}}
$$

Hence, we can assume $\mu_{1}=\mu_{2}=\mu$ and $\mu_{3}=-2 \mu$. Since $\sum_{i} \mu_{i}^{3}=-6 \mu^{3}$, we have

$$
\mu=-\frac{\sqrt{B}}{\sqrt{6}}<0
$$

For any real numbers $a>0, x$ and $y$, the following inequality holds:

$$
2 x y \leq a x^{2}+\frac{1}{a} y^{2} .
$$

By making use of the inequality (3.42) and Lemma 3.8, we have at $p$

$$
\begin{aligned}
-\frac{1}{2}\left(A_{1}-A_{2}\right)^{2} \leq & \frac{39 A_{1}^{2}-32 A_{1} A_{2}-48 A_{2}^{2}}{18}+\left(\frac{9}{2} B \mu+3 r \mu^{2}\right) A_{1} \\
& +\left(\frac{2}{9} r-\frac{10 \mu}{3}\right) B A_{2}-\left(\frac{3}{8} r+\frac{9}{8} \mu\right) \mu^{2}\left(6 A_{1}+4 A_{2}\right) .
\end{aligned}
$$

This implies

$$
\begin{aligned}
0 \leq & \frac{48 A_{1}^{2}-50 A_{1} A_{2}-39 A_{2}^{2}}{18}+\left(\frac{9}{2} B \mu+3 r \mu^{2}\right) A_{1} \\
& +\left(\frac{2}{9} r-\frac{10 \mu}{3}\right) B A_{2}-\left(\frac{3}{8} r+\frac{9}{8} \mu\right) \mu^{2}\left(6 A_{1}+4 A_{2}\right) .
\end{aligned}
$$

From (3.7), we have at $p$

$$
\begin{aligned}
& \frac{-48 A_{1}^{2}+50 A_{1} A_{2}+39 A_{2}^{2}}{18} \\
& =\frac{-8\left(6 A_{1}+4 A_{2}\right) A_{1}+\frac{39}{4}\left(6 A_{1}+4 A_{2}\right) A_{2}+\frac{47}{2} A_{1} A_{2}}{18} \\
& =-\frac{4}{3} B\left(\mu-\frac{r}{6}\right) A_{1}+\frac{13}{8} B\left(\mu-\frac{r}{6}\right) A_{2}+\frac{47}{36} A_{1} A_{2} .
\end{aligned}
$$

Therefore, (3.43) and (3.44) yield the following at $p$

$$
0 \leq-\frac{47}{36} A_{1} A_{2}+\left(\frac{113}{24} \mu-\frac{7}{72} r\right) B A_{1}+\left(-\frac{137}{24} \mu+\frac{35}{144} r\right) B A_{2}
$$


Since $\mu<0, r<0$ and $\frac{r^{2}}{3}+\left(\frac{7}{113}\right)^{2} \frac{2 r^{2}}{3}<S<\frac{r^{2}}{3}+\left(\frac{35}{137}\right)^{2} \frac{r^{2}}{6}$, then $\frac{113}{24} \mu-\frac{7}{72} r<0$ and $-\frac{137}{24} \mu+\frac{35}{144} r<0$ hold. From $A_{1} \geq 0, A_{2} \geq 0, B>0$, we infer $A_{1}=A_{2}=0$. But (3.7) and $A_{1}=A_{2}=0$ say that $B=0$ or $\mu=\frac{r}{6}$. This is impossible. Hence, we can conclude that Theorem 3.0 holds. Thus we have completed the proof of Theorem 3.0.

Proof of Main Theorem. If we suppose that there exists a complete locally conformally flat 3-dimensional Riemannian manifold which satisfies the conditions in the Main Theorem, from the Theorem 3 in the Section 2, we have

$$
\sup \sum_{i=1}^{3} \mu_{i}^{3}=\frac{B^{\frac{3}{2}}}{\sqrt{6}}
$$

From the proof of Theorem 3 in [1], we know that there exists a sequence $\left\{p_{m}\right\}$ of points in $M^{3}$ such that

$$
\lim _{m \rightarrow \infty} \sum_{i=1}^{3} \mu_{i}^{3}\left(p_{m}\right)=\sup \sum_{i=1}^{3} \mu_{i}^{3}=\frac{B^{\frac{3}{2}}}{\sqrt{6}} .
$$

Since $B$ is constant, we have that $\mu_{i}$ for any $i$ is bounded. From formulas (2.3) and (2.4) in Proposition, we know that $R_{i j, k}$ and $R_{i j, k l}$ are bounded for any $i, j, k$ and $l$. Thus, by taking a subsequence if necessary, we can assume $\lim _{m \rightarrow \infty} \mu_{i}\left(p_{m}\right)=\mu_{i}^{*}$, $\lim _{m \rightarrow \infty} R_{i j, k}\left(p_{m}\right)=R_{i j, k}^{*}$ and $\lim _{m \rightarrow \infty} R_{i j, k l}\left(p_{m}\right)=R_{i j, k l}^{*}$. Since $\sum_{i}\left(\mu_{i}^{*}\right)^{3}=$ $\sup \sum_{i} \mu_{i}^{3}=\frac{B^{\frac{3}{2}}}{\sqrt{6}}$ holds if and only if at least two of $\mu_{i}^{*}$ are equal. Hence, we can put $\mu_{1}^{*}=\mu_{2}^{*}=\mu$ and $\mu_{3}^{*}=-2 \mu$. By replacing the point $p$ which was used in the proofs of Lemmas and Theorem 3.0 with the above limit and making use of the same proofs, we can conclude that the Main Theorem holds. In fact, although we do not know whether $\lim _{m \rightarrow \infty} R_{i j, k l t}\left(p_{m}\right)=R_{i j, k l t}^{*}$ holds, from Ricci formulas in the Section 2, we know that $\lim _{m \rightarrow \infty}\left(R_{i j, k l t}-R_{i j, k t l}\right)\left(p_{m}\right)$ exists. From the proof of Lemma 3.5, we can infer $\lim _{m \rightarrow \infty} \sum_{i, j, k} R_{i j, k} R_{i j, k 33}\left(p_{m}\right)$ exists. Therefore, we have $\lim _{m \rightarrow \infty} \sum_{i, j, k} R_{i j, k} R_{33, i j k}\left(p_{m}\right)$ also exists because $\lim _{m \rightarrow \infty} \sum_{i, j, k} R_{i j, k}\left(R_{33, i j k}-\right.$ $\left.R_{i j, k 33}\right)\left(p_{m}\right)$ exists. Thus, formula (3.18) in the proof of Lemma 3.6 can also be obtained when we replace the point $p$ with the above limit. Making use of these facts above, these Lemmas in the section can be proved by the same methods. Hence, we can conclude that the Main Theorem is true.

\section{References}

[1] Q.M. Cheng, S. Ishikawa and K. Shiohama, Conformally flat 3-manifolds with constant scalar curvature, J. Math. Soc. Japan, 51 (1999), 209-226.

[2] R. Schoen, Conformal deformation of a Riemannian metric to constant scalar curvature, J. Differential Geometry, 20 (1984), 479-495. 
[ 3 ] R. Schoen and S.T. Yau, Conformally flat manifolds, Kleinian groups, and constant scalar curvature, Invent. Math., 92 (1988), 47-71.

[4] R. Schoen and S.T. Yau, Differential Geometry, Science Press, Beijing, 1988.

[5] N. Trudinger, Remarks concerning the conformal deformation of Riemannian structures on compact manifolds, Ann. Scuola Norm. Sup. Pisa, Cl. Sci., 22 (1968), 265-274.

[6] H. Yamabe, On the conformal deformation of Riemannian structures on compact manifolds, Osaka J. Math., 12 (1960), 21-37.

\author{
QING-MING CHENG \\ Department of Mathematics \\ Faculty of Science \\ JOSAI UNIVERSITY \\ SAITAMA, SAKado 350-0295, JAPAN \\ Susumu IsHIKaWA \\ Department of Mathematics \\ SAGA UNIVERSITY \\ SAGA 840-0027, J APAN \\ Katsuhiro Shiohama \\ Department of Mathematics \\ SAGA UNIVERSITY \\ SAGA 840-0027, JAPAN
}

